X. WU, H.-C. LIN, M.-L. LI, L.-L. LI, Z.-Y. HAN, L.-Z. GONG* (UNIVERSITY OF SCIENCE AND TECHNOLOGY OF CHINA, HEFEI AND COLLABORATIVE INNOVATION CENTER OF CHEMICAL SCIENCE AND ENGINEERING, TIANJIN, P. R. OF CHINA)

Enantioselective 1,2-Difunctionalization of Dienes Enabled by Chiral Palladium Complex-Catalyzed Cascade Arylation/Allylic Alkylation Reaction

J. Am. Chem. Soc. 2015, 137, 13476-13479.

\title{
Category
}

Metal-Catalyzed Asymmetric

Synthesis and

Stereoselective

Reactions

\section{Palladium-Catalyzed Enantioselective 1,2-Difunctionalization of 1,3-Dienes}

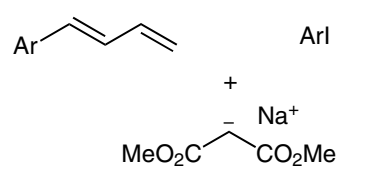

Selected examples:<smiles>CC(=O)C(C=Cc1ccc(C)cc1)CC(Cc1ccc(C)cc1)C(C)=O</smiles>

$71 \%$ yield

$86 \%$ ee

regioselectivity $\geq 15: 1$

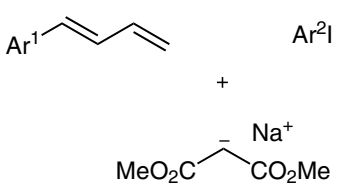

Selected examples:

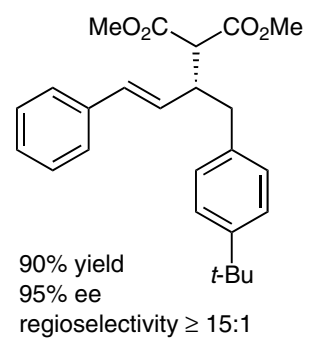

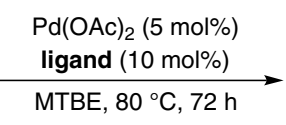

major product<smiles>COC(=O)C(Br)C(=CCCBr)C(C)=O</smiles>

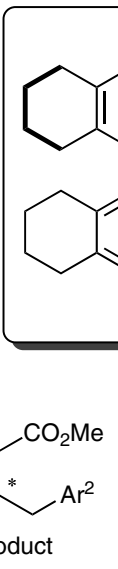

d(OAc) $)_{2}(5 \mathrm{~mol} \%)$ ligand $(10 \mathrm{~mol} \%)$ MTBE, $80^{\circ} \mathrm{C}, 72 \mathrm{~h}$<smiles>CC(=O)C(Cc1ccccc1C(F)(F)F)[C@H](/C=C/c1ccccc1)C(C)=O</smiles>

$74 \%$ yield

$98 \%$ ee

regioselectivity $\geq 15: 1$<smiles>COC(=O)C(C=Cc1ccccc1Cl)[C@@H](C=Cc1ccccc1Cl)C(C)=O</smiles>

$62 \%$ yield

$94 \%$ ee

regioselectivity $\geq 15: 1$

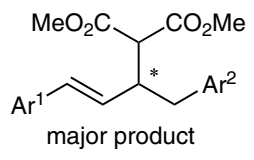<smiles>COC(=O)C(Br)C(=CCCBr)C(C)OC</smiles><smiles>COC(=O)C(CBr)[C@@H](/C=C/[Al])CCBr</smiles><smiles>COC(=O)C([17Br])[C@@H](/C=C/C[Al])C(C)=O</smiles><smiles>COC(=O)C(C=Cc1cccs1)C(Cc1ccccc1)Cc1ccccc1</smiles>

$83 \%$ yield

$92 \%$ ee

regioselectivity $=9: 1$

\section{Key words}

\section{dienes}

difunctionalization

palladium

asymmetric

catalysis

cascade reaction

arylation

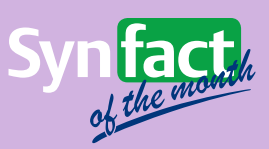

Significance: The authors report a palladiumcatalyzed enantioselective three-component coupling of 1,3-dienes with aryl iodides and sodium dialkylmalonates by using a $\mathrm{H}_{8}$-BINOL-based phosphoramidite ligand. A series of chiral 1,2-difunctionalized products were prepared in good yields ( $\leq 93 \%)$ with high regio- and enantioselectivities (15:1 or better and $\leq 98 \%$ ee).
Comment: This reaction proceeds by a palladium-catalyzed cascade arylation and asymmetric allylic alkylation reaction, which provides an important alternative strategy for the enantioselective difunctionalization of 1,3-dienes, leading to synthetically useful chiral chemicals.

SYNFACTS Contributors: Hisashi Yamamoto, Masahiro Sai 